\title{
CORONAL ALFVÉN SPEED DETERMINATION: CONSISTENCY BETWEEN SEISMOLOGY USING AIA/SDO TRANSVERSE LOOP OSCILLATIONS AND MAGNETIC EXTRAPOLATION
}

\author{
E. Verwichte ${ }^{1,2}$, T. VAn Doorsselaere ${ }^{2}$, C. Foullon $^{1}$, And R.S. White ${ }^{1}$ \\ Draft version February 11, 2013
}

\begin{abstract}
Two transversely oscillating coronal loops are investigated in detail during a flare on the $6^{\text {th }}$ September 2011 using data from the Atmospheric Imaging Assembly (AIA) on board the Solar Dynamics Observatory (SDO). We compare two independent methods to determine the Alfvén speed inside these loops. Through the period of oscillation and loop length information about the Alfvén speed inside each loop is deduced seismologically. This is compared with the Alfvén speed profiles deduced from magnetic extrapolation and spectral methods using AIA bandpass. We find that for both loops the two methods are consistent. Also, we find that the average Alfvén speed based on loop travel time is not necessarily a good measure to compare with the seismological result, which explains earlier reported discrepancies. Instead, the effect of density and magnetic stratification on the wave mode has to be taken into account. We discuss the implications of combining seismological, extrapolation and spectral methods in deducing the physical properties of coronal loops.
\end{abstract}

Subject headings: MHD, Sun: corona, magnetic fields, oscillations

\section{INTRODUCTION}

The solar corona and the structures therein, such as loops, support magnetohydrodynamic (MHD) waves of various kinds (Deforest \& Gurman 1998; Aschwanden et al. 1999; Berghmans \& Clette 1999; Kliem et al. 2002; Verwichte et al. 2005; Tomczyk et al. 2007). Transverse waves, and in particular transverse loop oscillations (TLOs) have received much attention because they are manifestations of waves supported by the magnetic field and plasma structuring of the corona (Aschwanden et al. 1999; Nakariakov et al. 1999; Verwichte et al. 2004; Tomczyk et al. 2007; McIntosh et al. 2011). The synoptic, full-disk nature of AIA on board SDO (Lemen et al. 2012) observations confirm that they are ubiquitous and are present in many eruptive events (White \& Verwichte 2012). TLOs occur in loops of all sizes and temperatures, have periods spanning a few to tens of minutes (e.g. Aschwanden et al. 2002; Verwichte et al. 2010; White \& Verwichte 2012; White et al. 2012).

The comparison of the observed wave quantities with MHD wave theory provides a seismological route to determining local physical parameters that are difficult to measure directly (e.g. Verwichte et al. 2006b; Arregui et al. 2007; Goossens et al. 2008). Nakariakov \& Ofman (2001) demonstrated that by measuring the period and wavelength of the oscillation an estimate of the loop's Alfvén speed and local magnetic field strength can be obtained. Knowledge of those physical parameters is important for modelling dynamics in the solar corona such as solar flares and CMEs. Various authors have reported phase speeds typically of the order of $1000 \mathrm{kms}^{-1}$. The determination of the Alfvén speed from the observed phase speed lies at the heart of the seismological method

\footnotetext{
Electronic address: Erwin.Verwichte@warwick.ac.uk

${ }^{1}$ Centre for Fusion, Space and Astrophysics, Department of Physics, University of Warwick, Coventry CV4 7AL, UK

2 Centre for Plasma Astrophysics, Department of Mathematics, Katholieke Universiteit Leuven, Celestijnenlaan 200B, 3001 Leuven, Belgium
}

and employs the wave theory for kink modes. The basic theory by Edwin \& Roberts (1983) applies to internally uniform loops. However, to improve accuracy, effects such as density and magnetic stratification can be incorporated (Andries et al. 2005, 2009). In all versions of the wave theory, knowledge of the density contrast between the loop and the external corona is required to find the Alfvén speed. However, the exact value of the density contrast is difficult to measure directly (e.g. Aschwanden et al. 2003; Schmelz et al. 2003; Terzo \& Reale 2010).

Aschwanden \& Schrijver (2011) were the first to compare a seismologically determined magnetic field with a potential magnetic extrapolation. The seismologically determined magnetic field strength had been extracted using the density measured using a DEM method applied to the AIA bandpasses (Aschwanden et al. 2011; Hannah \& Kontar 2012). They found that it the average extrapolated magnetic field strength in the loop exceeded the seismologically determined value by a factor of three. We wish to establish the physical reasons for such a discrepancy. Because the loop's magnetic field strength is derived from the Alfvén speed by using the loop density (deduced from spectral measurement or from assumption), the Alfvén speed is the true seismologically determined physical loop quantity. We shall therefore determine the Alfvén speed from a new TLO event using AIA data and compare it with the Alfvén speed obtained by spectral and magnetic extrapolation methods. This has become feasible because of the superior quality of the AIA instrument and the multiple viewpoints made possible by the Extreme UltraViolet Imager (EUVI) on board STEREO (Howard et al. 2008; Wuelser et al. 2004; Verwichte et al. 2009).

The paper is organised as follows. In Sect. 2, we present the wave analysis two TLOs seen by AIA. In Sect. 3 the Alfvén speed using the 'direct' methods of magnetic extrapolation and DEM inversion is calculated. In Sect. 4 the role of stratification is examined in determining the 

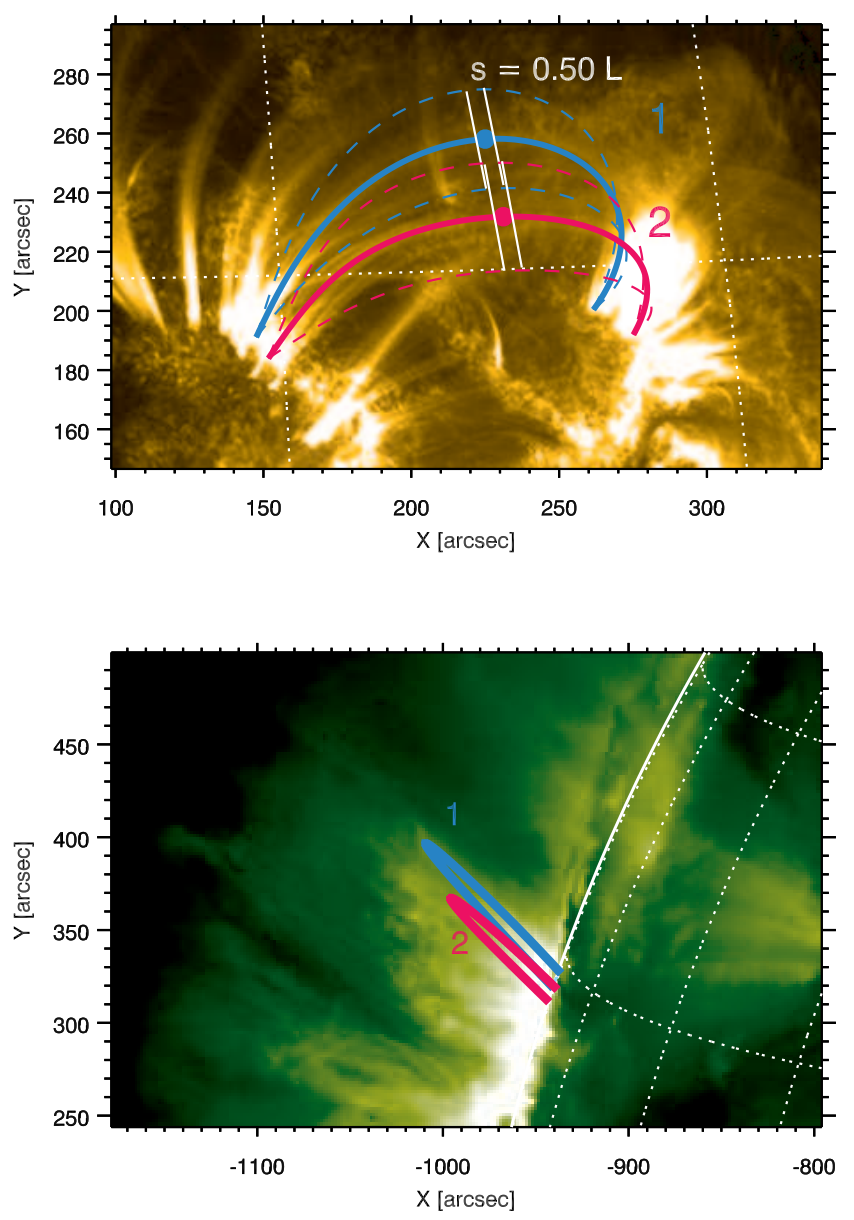

FIG. 1. - Detailed view of the loops at 22:15:30 UT in AIA/SDO $171 \AA$ (upper) and in EUVI/STEREO-A $171 \AA$ (lower).

equivalent Alfvén speed to compare with wave observations. In Sect. 5, the results from the comparison are used to determine the loop cross-section profile. Finally, in Sect 6 we discuss our findings, explore its potential and limitations.

\section{TRANSVERSE LOOP OSCILLATION EVENT}

We examine two loops in NOAA active region 11283 on the $6^{\text {th }}$ of September 2011, which exhibit transverse oscillations of periods around 2-3 minutes in response to a GOES class X2.1 flare inside the region starting at 22:12 UT and peaking at 22:20 UT. The location of these loops as seen by AIA/SDO in the $171 \AA$ bandpass in shown in Fig. 1. Following the technique detailed in Verwichte et al. (2010) and White \& Verwichte (2012) the loops are compared with their view in images from EUVI/STEREO-A $171 \AA$ at 22:15 UT to determine the general 3d geometry (see Fig. 1). STEREO-A is ahead of Earth by $103^{\circ}$ in longitude. To the projected loop path a third coordinate is added under the assumption that the whole loop lies within a plane. The inclination of that plane with respect to the photospheric normal, $\theta$, is now the only free parameter. The model loop is then transformed to the second viewpoint and $\theta$ is adjusted visually to best match the loop seen there. The threedimensional loop geometry gives loop lengths of $213 \mathrm{Mm}$
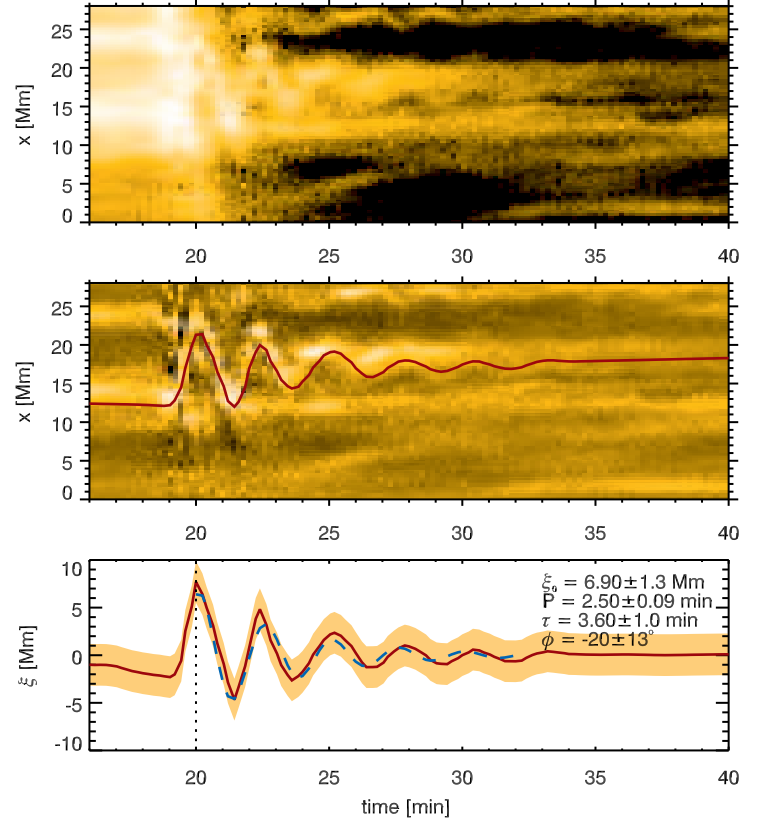

FIG. 2. - time-distance image of full intensity for the path at $s=0.5 \mathrm{~L}$. Time is counted in minutes from reference time 22:00 UT on 6 Sep 2011. Middle: Filtered time-distance image with the loop position of loop \#1 of Event A indicated. Bottom: displacement time series $\xi(t)$. The dashed line is a fitted damped sinusoidal curve. The dotted line indicated the start time used for the fit.

and $188 \mathrm{Mm}$, respectively. The loop geometry parameters are listed in Table 1.

We employ the tried and tested analysis technique for transverse oscillations that has been perfected by several studies (Verwichte et al. 2004; Van Doorsselaere et al. 2007; Verwichte et al. 2009, 2010; White \& Verwichte 2012). A rectangular box region of interest (data cut) is chosen that runs across the loop top in the direction aligned with the projected displacement of a hypothetical horizontally polarised TLO. The AIA time series are then interpolated at the ROI locations. For each time, we average the data over the 11-pixel width of the box to increase the signal-to-noise ratio. Thus, the dataset is reduced to a two-dimensional time-space image set containing the spatial average intensity along the ROI as a function of time. A TLO would appear as a periodic displacement of the loop location. To obtain the loop axis displacement, we fit a Gaussian shape (Carcedo et al. 2003) superimposed on a linear trend. The width of the fitted Gaussian profile is taken to be a good measure of error on the position. Finally, from the position a trend is subtracted and the loop's oscillation displacement time series, $\xi(t)$, is obtained, which is characterised by fitting a damped cosine function of the form $\xi(t)=\xi_{0} \exp \left[-\left(t-t_{0}\right) / \tau\right] \cos \left[2 \pi\left(t-t_{0}\right) / P+\phi\right]$ using a Levenburg-Marquardt least squares fitting method Markwardt (2009). The fitting parameters are the displacement amplitude, $\xi_{0}$, oscillation period, $P$, damping time, $\tau$, phase $\phi$ and reference time, $t_{0}$. The oscillation parameters are listed in Table 1.

The phase speed, $V_{\mathrm{ph}}$, is calculated as the ratio of the wavelength (for fundamental standing mode $\lambda=2 L$ ), over the period, where we allow for an error of typically $10 \%$ in $L$. For the two loops, we find phase speeds in the 
range $2500-2600 \mathrm{kms}^{-1}$. By interpreting the TLO as a Alfvénic kink mode in a thin, cylindrical loop uniform in the longitudinal direction and in the zero plasma- $\beta$ limit, the phase speed is equal to the kink speed, $C_{\mathrm{K}}$, given by (Edwin \& Roberts 1983):

$$
C_{\mathrm{K}} \approx \sqrt{\frac{2}{1+\zeta^{-1}}} V_{\mathrm{A}},
$$

where $\zeta=\rho_{0 i} / \rho_{0 e}$ is the ratio of internal to external densities and $V_{\mathrm{A}}$ is the Alfvén speed in the loop. We can thus calculate from the phase speed the value range of the loop Alfvén speed,

$$
V_{\mathrm{A}, \mathrm{s}}=V_{\mathrm{ph}} \sqrt{\frac{1+\zeta^{-1}}{2}},
$$

considering the two extreme values of $\zeta$, namely unity and infinity for an overdense loop. The exact value of the density contrast is difficult to measure (e.g. Aschwanden et al. 2003; Schmelz et al. 2003; Terzo \& Reale 2010). Expression (2) assumes that the loop is uniform with the internal Alfvén speed equal to $V_{\mathrm{A}, \mathrm{s}}$ all along the loop. However, it is expected that the loops are stratified in density and magnetic field strength and that the Alfvén speed varies along the loop. Aschwanden \& Schrijver (2011) equate $V_{\mathrm{A}, \mathrm{s}}$ with the average speed in the loop for to the same travel time.

\section{COMPARISON BETWEEN SEISMOLOGICAL AND DIRECT DETERMINATION OF ALFVÉN SPEED}

The Alfvén speed $V_{\mathrm{A}, \mathrm{s}}$ can be exploited seismologically to determine the loop magnetic field strength (Nakariakov \& Ofman 2001). However, in order to do so the value of the loop density is required. Often in absence of spectroscopic information of the loop density, and employing the argument that the density appears only weakly (through a square root) in the Alfvén speed, a plausible value of the electron number density $n_{e}=10^{15}$ $\mathrm{m}^{-3}$ had been assumed (Verwichte et al. 2004). For this density, we find values for the magnetic field strength for each loop in the ranges 25-46 $\mathrm{G}$ and $25-48 \mathrm{G}$, respectively. If we assume a density one order of magnitude smaller, we find values of 8-15 G for both loops instead. We shall attempt to test the consistency of the seismological method by estimating the magnetic field and loop density independently. We can then compare the average Alfvén speeds from both methods.

First, the magnetic field is determined using the Potential Field Source Surface (PFSS) potential field extrapolation tool (Schrijver 2001; Schrijver \& De Rosa 2003), which produces a data cube of all three components of magnetic field in spherical coordinates. Figure 3 shows the potential field extrapolation of the active region. The projected loop paths are indicated in red. The magnetic field is interpolated for the three-dimensional loop paths. We can see that the paths approximately align with the potential field, but not fully. Misalignments with an angle between 20 and 40 degrees between potential-field extrapolations and loops have been reported in the past (Sandman et al. 2009). The average ratio of parallel to total magnetic field strength is $60 \%$. Importantly, for our study only the field strength is required, which is expected to be less sensitive. The top panels in Figures 4 and 5 shows the magnetic field strength along the loop.
TABLE 1

\begin{tabular}{|c|c|c|}
\hline Quantities & Loop \#1 & Loop \#2 \\
\hline \multicolumn{3}{|l|}{ Loop geomety } \\
\hline Loop length $L$ & $188 \pm 20 \mathrm{Mm}$ & $160 \pm 20 \mathrm{Mm}$ \\
\hline Loop inclination angle $\theta$ & $27^{\circ} \mathrm{N}$ & $25^{\circ} \mathrm{N}$ \\
\hline Loop height $h$ & $64 \mathrm{Mm}$ & $49 \mathrm{Mm}$ \\
\hline Footpoint separation $\Delta \alpha$ & $7.1^{\circ}$ & $7.6^{\circ}$ \\
\hline \multicolumn{3}{|l|}{ Oscillation parameters } \\
\hline Oscillation period $P$ & $150 \pm 5, \mathrm{~s}$ & $122 \pm 6, \mathrm{~s}$ \\
\hline Damping time $\tau$ & $216 \pm 60, \mathrm{~s}$ & $348 \pm 400, \mathrm{~s}$ \\
\hline Displacement amplitude $\xi_{0}$ & $6.9 \pm 1 \mathrm{Mm}$ & $1.9 \pm 1 \mathrm{Mm}$ \\
\hline Phase $\phi$ & $340 \pm 10^{\circ}$ & $299 \pm 30^{\circ}$ \\
\hline Reference time $t_{0}$ & $22: 04 \mathrm{UT}$ & $22: 04 \mathrm{UT}$ \\
\hline Mode number $n$ & 1 & 1 \\
\hline Wavelength $\lambda$ & $380 \pm 40 \mathrm{Mm}$ & $320 \pm 30 \mathrm{Mm}$ \\
\hline Phase speed $V_{\mathrm{ph}}$ & $2510 \pm 400 \mathrm{~km} / \mathrm{s}$ & $2620 \pm 400 \mathrm{~km} / \mathrm{s}$ \\
\hline Seis. Alfvén speed $V_{\mathrm{A}, \mathrm{s}}$ & $1780-2510 \mathrm{~km} / \mathrm{s}$ & $1860-2620 \mathrm{~km} / \mathrm{s}$ \\
\hline \multicolumn{3}{|l|}{ Extrapolated quantities } \\
\hline Av. magn. field $\langle B>$ & $26 \mathrm{G}$ & $41 \mathrm{G}$ \\
\hline Weig. magn. field $<B_{\mathrm{W}}>$ & $19 \mathrm{G}$ & $32 \mathrm{G}$ \\
\hline Footpoint density $n_{e, \mathrm{fp}}$ & $0.710^{15} \mathrm{~m}^{-3}$ & $0.710^{15} \mathrm{~m}^{-3}$ \\
\hline Density scaleheight $H$ & $65 \mathrm{Mm}$ & $139 \mathrm{Mm}$ \\
\hline Loop temperature $T_{0}$ & $0.79 \mathrm{MK}$ & $0.79 \mathrm{MK}$ \\
\hline Loop minor radius $a$ & $0.95 \mathrm{Mm}$ & $0.85 \mathrm{Mm}$ \\
\hline Alfvén speed scaleheight $\Lambda$ & $107 \mathrm{Mm}$ & $98 \mathrm{Mm}$ \\
\hline loop top Alfvén speed $V_{\mathrm{A}, \text { top }}$ & $1470 \mathrm{~km} / \mathrm{s}$ & $1640 \mathrm{~km} / \mathrm{s}$ \\
\hline Av. Alfvén speed $\left\langle V_{\mathrm{A}}\right\rangle$ & $2960 \mathrm{~km} / \mathrm{s}$ & $3430 \mathrm{~km} / \mathrm{s}$ \\
\hline Weig. Alfvén speed $<V_{\mathrm{A}, \mathrm{W}}>$ & $2260 \mathrm{~km} / \mathrm{s}$ & $2700 \mathrm{~km} / \mathrm{s}$ \\
\hline Dir. Alfvén speed $V_{\mathrm{A}, \mathrm{d}}$ & $2130 \mathrm{~km} / \mathrm{s}$ & $2480 \mathrm{~km} / \mathrm{s}$ \\
\hline \multicolumn{3}{|l|}{ Loop cross - sectional profile } \\
\hline Inverse density contrast $\zeta^{-1}$ & $0.4(0.1-0.9)$ & $0.8(0.3-1)$ \\
\hline Transition layer thickness $\ell / a$ & $1.1(0.6-2)$ & $1.9(0-2)$ \\
\hline
\end{tabular}

PHYSICAL LOOP QUANTITIES

We estimate the density of the loops from the AIA bandpasses following a method outlined in Aschwanden \& Schrijver (2011). However, as the loops are only seen clearly in the $171 \AA$ bandpass, we assume that the temperature of the loop corresponds to the peak temperature of that bandpass. The observed intensity, normalised with exposure time, is related to the differential emission measure as

$$
I_{171}=\int_{0}^{\infty} D E M(T) R_{171}(T) \mathrm{d} T,
$$

where $R_{171}(T)$ is the response function for the $171 \AA$ bandpass. The contribution to the intensity from the loop itself is determined from fitting at each position along the loop a Gaussian profile with background. Assuming an isothermal plasma, the DEM becomes trivially

$$
\operatorname{DEM}(T)=n_{e}^{2} \operatorname{LOS} \delta\left(T-T_{0}\right)
$$




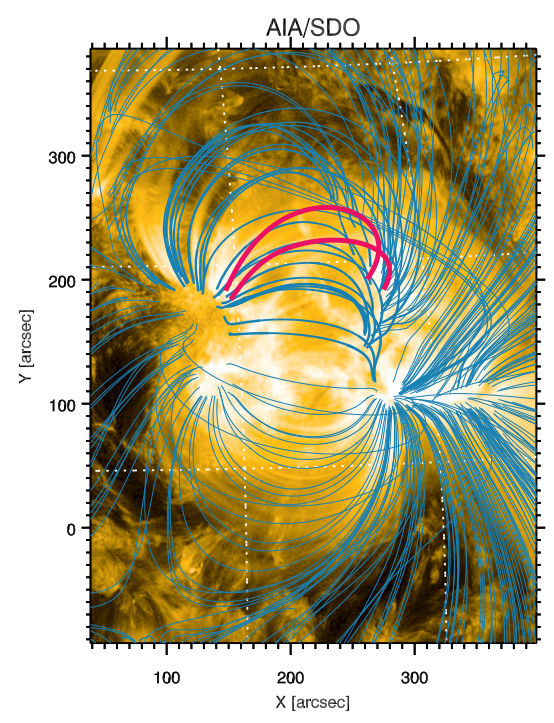

FIG. 3.- PFSS potential field extrapolation of active region NOAA 11283 on 7 Sep 2011 at 00:00 UT. The two oscillating loops are indicated in red.

where $n_{e}$ is the electron number density, $\operatorname{LOS}(s)$ is the distance across the loop, which takes into account the geometry of the loop with respect to the line-of-sight direction, and $T_{0}=0.79 \mathrm{MK}$ is the peak temperature of the $171 \AA$ bandpass. Then, the loop electron number density is found as

$$
n_{e}(s)=\sqrt{\frac{I_{\text {loop }}(s)}{R_{171}\left(T_{0}\right) L O S(s)}} .
$$

Because we assume the temperature corresponds to the peak temperature of the bandpass (i.e. $R_{171}\left(T_{0}\right)$ is maximal), the thus determined density may be regarded as a lower limit. Panels (c) of Figures 4 and 5 show the density as a function of distance along the loop. We find the density to vary between $10^{15}$ and $10^{15} \mathrm{~m}^{-3}$. The profile is noisy due to line-of-sight interference but a clear trend of decreasing density towards the loop top can be seen. We fit an exponential function to the density profile of the form

$$
n_{e}(s)=n_{e, \mathrm{fp}} \mathrm{e}^{-\frac{z(s)}{H}}
$$

where $z$ is the height above the photosphere and $H$ is the density scale-height. We find values for the density scale-height of $65 \mathrm{Mm}$ and $139 \mathrm{Mm}$, respectively. If we assume an isothermal loop, these would correspond to temperatures of 1.3 MK and 2.8 MK, respectively. The loops probably do not have these temperatures as they are not clearly visible in the $193 \AA$ and $211 \AA$ bandpasses, whose peak temperature is in this range. So-called superhydrostatic density scale heights have been determined before (e.g. Van Doorsselaere et al. 2007).

In panels (d) we combine the magnetic field strength and electron density and find the Alfvén speed as a function of distance along the loop as

$$
V_{\mathrm{A}}(s)=\frac{B(s)}{\sqrt{\mu_{0} \tilde{\mu} m_{p} n_{e}(s)}},
$$

where $\tilde{\mu}=1.2$ for coronal abundances. The average Alfvén speed across the loop, $\left\langle V_{\mathrm{A}}\right\rangle$, is defined as the
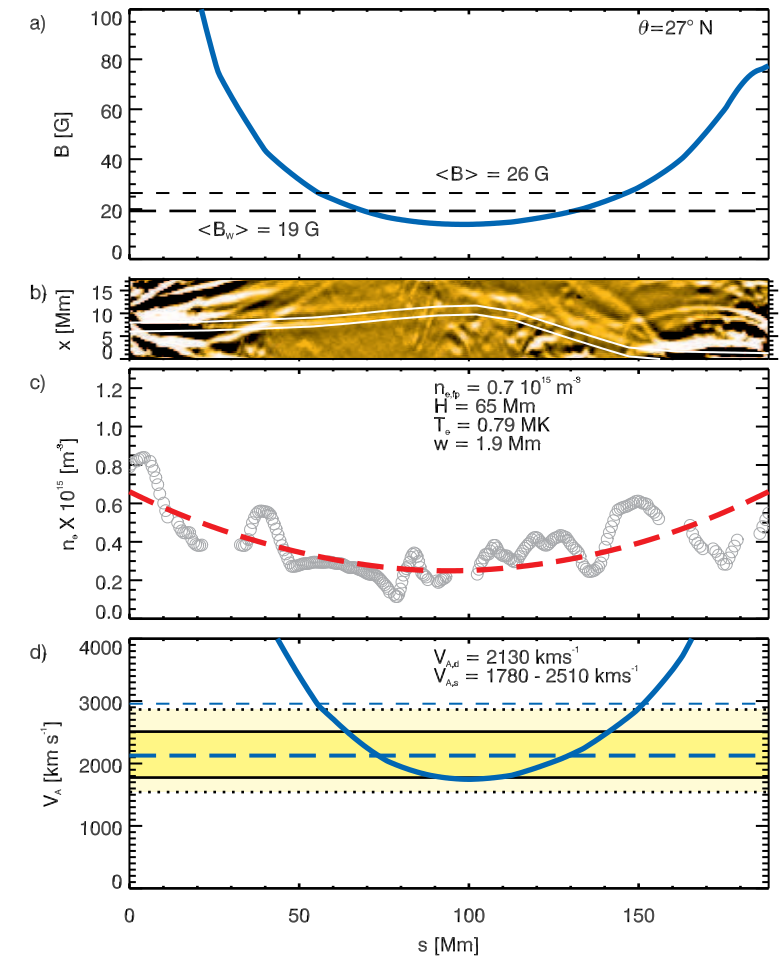

FIG. 4.- For loop \#1, a comparison of the Alfvén speed derived seismologically and derived using magnetic extrapolation with potential fields and spectroscopy using SDO/AIA bandpasses. a) Magnetic field strength from PFSS model at 7 Sep 2011 (00:00 UT), interpolated along the $3 \mathrm{~d}$ path of the loop. The magnetic field averages $\langle B\rangle$ and $\left\langle B_{W}\right\rangle$ are indicated as dashed and long-dashed lines, respectively. b) AIA 171 Aintensity along the path of the loop. The detailed location of the loop is indicated by two solid lines. c) Electron number density derived using Eq. (5) as a function of distance along the loop. The dashed line is a fit of the form (6). d) Alfvén speed as a function of distance along the loop. The thick blue curve is the Alfvén speed along the loop determined using Eq. (7). The horizontal shaded region is the range of values for the seismologically determined Alfvén speed, $V_{\mathrm{A}, \mathrm{s}}$, using Eq. (2) for an arbitrary value of the loop density contrast. The light shaded region bordered by dotted lines extends this range by including the measurement errors of loop length and oscillation period. The thin dashed line is the average Alfvén speed $\left\langle V_{\mathrm{A}}\right\rangle$. The thick blue dashed line is the Alfvén speed, $V_{\mathrm{A}, \mathrm{d}}$.

constant speed that gives the same travel time between the two foot points (Aschwanden \& Schrijver 2011):

$$
\int_{0}^{L} \frac{\mathrm{d} s}{V_{\mathrm{A}}(s)}=\Delta t=\frac{L}{<V_{\mathrm{A}}>} \Rightarrow<V_{\mathrm{A}}>=L\left[\int_{0}^{L} \frac{\mathrm{d} s}{V_{\mathrm{A}}(s)}\right]^{-1} .
$$

Figures 4 and 5 show that the average Alfvén speed exceeds the seismologically determined Alfvén speed range by up to a factor of two. This result is consistent with what was found by Aschwanden \& Schrijver (2011). It seems to indicate that there is a mismatch between seismological and direct methods in determining the loop Alfvén speed. Following the definition of the average Alfvén speed we calculate the average magnetic field as

$$
<B>=L\left[\int_{0}^{L} \frac{\mathrm{d} s}{B(s)}\right]^{-1}
$$



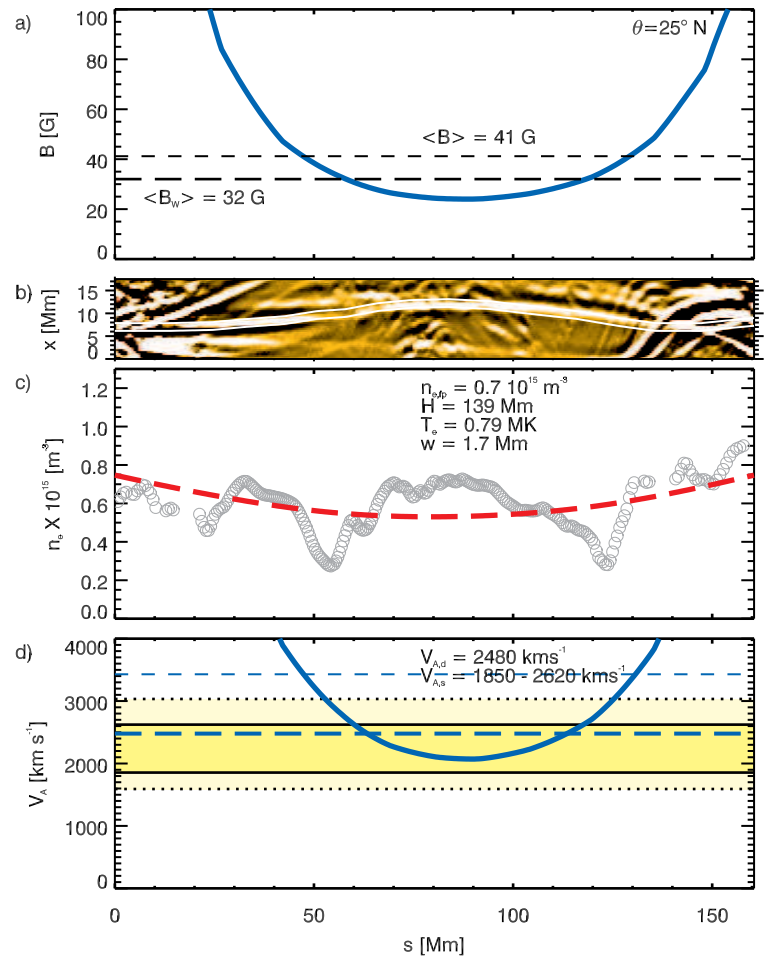

FIG. 5.- Same as Fig. 4 but for loop \#2.

where $B(s)$ is the magnetic field strength along the threedimensional loop path. For the two loops, we find that the magnetic field strength varies between more than 100 Gauss at the foot points to values around or below 20 Gauss at the loop tops. The average magnetic field strength is $26 \mathrm{G}$ and $41 \mathrm{G}$, respectively. These values fall within the range that was found seismologically with $n_{e}$ $\sim 10^{15} \mathrm{~m}^{-3}$.

We may improve the averaging by including a weight that takes into account the localisation of wave energy along the loop. Thus, the average Alfvén speed is modified to be

$$
<V_{\mathrm{A}, \mathrm{W}}>=L\left[\int_{0}^{L} \frac{W(s) \mathrm{d} s}{V_{\mathrm{A}}(s)}\right]^{-1},
$$

with weight function

$$
W(s)=n_{e}(s) \xi^{2}(s) L\left[\int_{0}^{L} n_{e}(s) \xi^{2}(s) \mathrm{d} s\right]^{-1} .
$$

$\xi(s)$ here is the mode displacement profile. When not using the mode profile from solving Eq. (12), it may be approximated using $\xi(s)=\xi_{0} \sin (\pi s / L)$. The average magnetic field may be weighted in the same manner. As Table 1 shows, the weighted average Alfvén speeds for the two loops lies closer to the seismological Alfvén speed range but is still represents an over-estimation.

\section{ROLE OF STRATIFICATION}

We wish to take the analysis a step further and consider the role of stratification on the TLO. The longitudinal structuring of the Alfvén speed will modify the

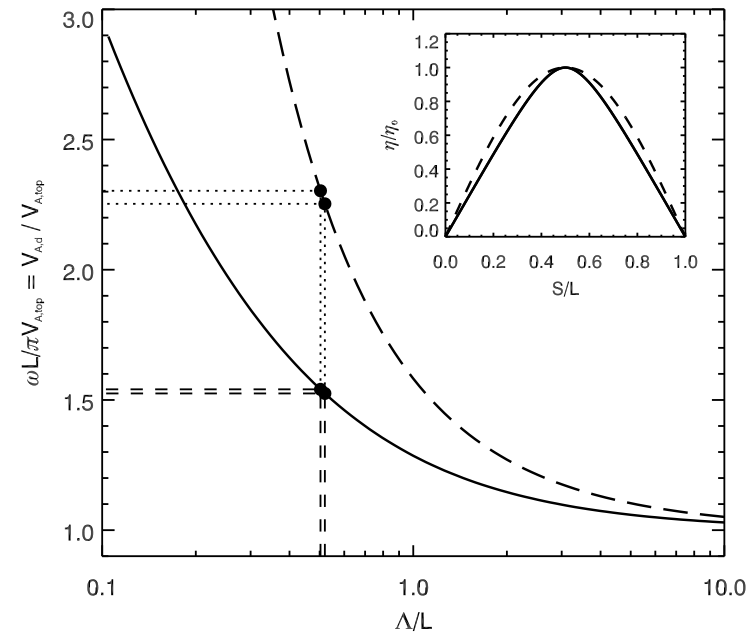

FIG. 6. - Normalised Alfvén speed $V_{\mathrm{A}, \mathrm{d}} / V_{\mathrm{A}, \text { top }}=\tilde{\omega} L / \mathrm{A}$,top as a function of normalised Alfvén speed scale height $\Lambda / L$ from solving Eq. (13). The long-dashed curve corresponds to the solution using the average Alfvén speed $\left\langle V_{\mathrm{A}}\right\rangle$. The values corresponding to the two loops are indicated. The inset shows the displacement profile along the loop. The dashed line is a sine curve.

oscillation period and phase speed (Andries et al. 2009). In the thin flux-tube limit, the spatial structure of a TLO may be modelled using the following differential equation (Dymova \& Ruderman 2005, 2006; Verth \& Erdélyi 2008)

$$
\frac{\mathrm{d}^{2} \eta(s)}{\mathrm{d} s^{2}}+\frac{\omega^{2}}{C_{\mathrm{K}}^{2}(s)} \eta(s)=0,
$$

where $\eta(s)=\xi / a$ is the transverse loop displacement relative to the local loop radius and $\omega$ is the mode frequency. Instead of modelling the kink speed in Eq. (12) using a range of values of $\zeta$, we take an alternate approach. We introduce $\tilde{\omega}=\sqrt{1+\zeta^{-1}} \omega / \sqrt{2}$. We assume that $\zeta$ is constant along the loop. Then Eq. (12) is modified to

$$
\frac{\mathrm{d}^{2} \eta}{\mathrm{d} s^{2}}+\frac{\tilde{\omega}^{2}}{V_{\mathrm{A}}^{2}(s)} \eta=0,
$$

From the resulting modified mode frequency, we define a phase speed, $V_{\mathrm{A}, \mathrm{d}}$, as

$$
V_{\mathrm{A}, \mathrm{d}}=\frac{\tilde{\omega} L}{\pi} .
$$

We have two approaches to solve Eq. (13). In the first approach, we solve Eq. (13) completely numerically using a Runga-Kutta algorithm with adaptive step size (Press et al. 2007). The mode frequency $\tilde{\omega}$ is then found using a shooting method. A second approach is to first solve Eq. (13) analytically by modelling the Alfvén speed profile with an exponential profile of the form $V_{\mathrm{A}}(s)=V_{\mathrm{A} \text {,top }}$ $\exp (|s-L / 2| / \Lambda)$ where $\Lambda$ represents a typical scale height of the Alfvén speed. Then, the solution of Eq. (13) with zero foot point displacement is in terms of Bessel functions of order 0 (Ferraro \& Plumpton 1958; McEwan et al. 2008)

$$
\eta(s)=\eta_{0}\left[\mathrm{Y}_{0}\left(k x_{\mathrm{L}}\right) \mathrm{J}_{0}(k x)-\mathrm{J}_{0}\left(k x_{\mathrm{L}}\right) \mathrm{Y}_{0}(k x)\right],
$$

where $k=\tilde{\omega} \Lambda / V_{\mathrm{A}, \text { top }}=(\Lambda \pi / L)\left(V_{\mathrm{A}, \mathrm{d}} / V_{\mathrm{A}, \text { top }}\right), x_{L}=$ $\exp (-L / 2 \Lambda)$ and $x=\exp (-|s-L / 2| / \Lambda)$. The wave frequency $\tilde{\omega}$, and through Eq. (14) also $V_{\mathrm{A}, \mathrm{d}}$, is then found 
as the fundamental mode solution of the dispersion relation

$$
\mathrm{Y}_{0}\left(k x_{\mathrm{L}}\right) \mathrm{J}_{1}(k)-\mathrm{J}_{0}\left(k x_{\mathrm{L}}\right) \mathrm{Y}_{1}(k)=0,
$$

which is solved numerically using e.g. a bracketing rootfinding algorithm. For the two loops using the Alfvén speed profiles from Figs. 4 and 5 we find fit values of $\Lambda=107 \mathrm{Mm}$ and $98 \mathrm{Mm}$, and $V_{\mathrm{A}, \text { top }}=1470 \mathrm{kms}^{-1}$ and $1640 \mathrm{kms}^{-1}$, respectively. The numerically derived values are listed in Table 1.

We have determined $V_{\mathrm{A}, \mathrm{d}}$ using both numerical and analytical approaches and they give equivalent results. Figures 4 and 5 show that for both loops $V_{\mathrm{A}, \mathrm{d}}$ (found with the numerical approach) falls inside the observed range of Alfvén speeds. This suggests that the seismological and direct methods are consistent.

It also show the importance of interpreting correctly the observed Alfvén speed range. Our findings indicate that the average Alfvén speed $\left\langle V_{\mathrm{A}}\right\rangle$ is not necessarily a good measure to compare with the seismology. Figure 6 shows the dependency of $\tilde{\omega}$ on $\Lambda$ and this may be used to quickly read the solution for given $\Lambda$ without need of explicitly solving Eq. (13). The solution is fitted by a power-law of the form

$$
V_{\mathrm{A}, \mathrm{d}}=V_{\mathrm{A}, \mathrm{top}}\left[1+0.27\left(\frac{\Lambda}{L}\right)^{-0.93}\right] \text {. }
$$

Figure 6 also clearly illustrates the disparity between the full solution and the use of the average Alfvén speed, which becomes significant for $\Lambda \lesssim L$. In the cases studies, $\Lambda / L \approx 0.5$ and $\left\langle V_{\mathrm{A}}>\right.$ is nearly 1.5 times as large as $V_{\mathrm{A}, \mathrm{d}}$.

\section{DETERMINATION OF THE LOOP CROSS-SECTIONAL PROFILE}

If we equate the Alfvén speed $V_{\mathrm{A}, \mathrm{s}}$ in Eq. (2) with $V_{\mathrm{A}, \mathrm{d}}$, then we can directly calculate the density contrast $\zeta$, i.e.

$$
\zeta^{-1}=2\left(\frac{V_{\mathrm{A}, \mathrm{d}}}{V_{\mathrm{ph}}}\right)^{2}-1,
$$

assuming $\zeta$ is constant along the loop. For loops \#1 and $\# 2$ we find values of $\zeta^{-1}=0.4$ and $\zeta^{-1}=0.8$, respectively. Note that the uncertainties are large. We may obtain seismologically more information about the loop cross-section by including the observed damping rate. The leading theory that explains the rapid damping of the oscillations is resonant absorption. It critically depends on the thickness of a thin transition layer, $\ell$, over which the density drops from inner to external conditions. Under the assumption of small thickness, $\ell \ll a$, it is given by (e.g. Ionson 1978; Hollweg \& Yang 1988; Goossens et al. 1992; Ruderman \& Roberts 2002)

$$
\frac{\ell}{a}=F \frac{\zeta+1}{\zeta-1} \frac{P}{\tau} .
$$

where $\ell / a$ is between 0 and 2 . For a half-wavelength sinusoidally varying density profile across the layer, $F=2 / \pi$ (Ruderman \& Roberts 2002). Equation (19) is strictly speaking only in the regime where $\ell \ll a$, though it still provides a relatively accurate extension into the regime of finite resonance layer widths (Van Doorsselaere et al. 2004). Also, Eq. (19) does not describe any transient

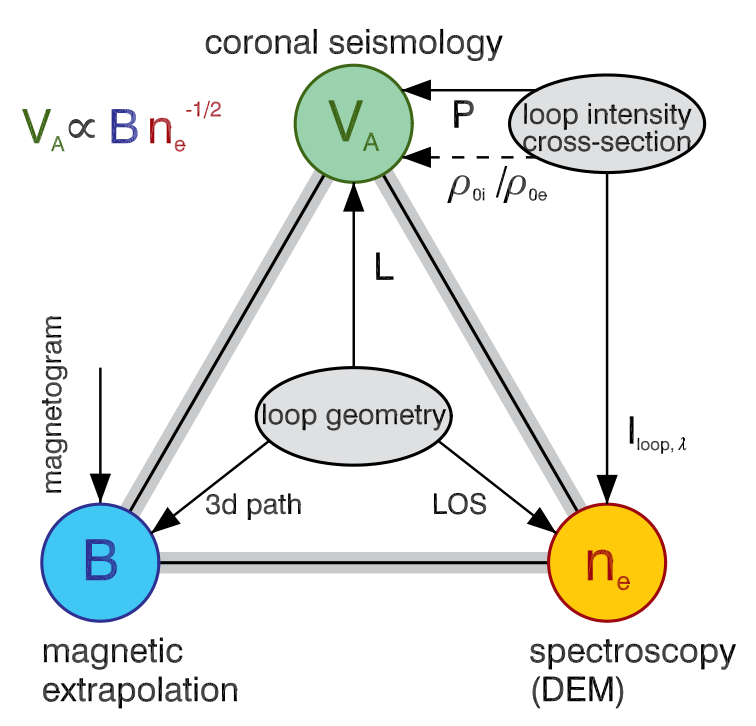

FIG. 7.- Principle how the techniques of coronal seismology, magnetic extrapolation and spectroscopy and their determination of the Alfvén speed, magnetic field strength and density are linked with each other through the formula of the Alfvén speed.

behaviour in the damping (Pascoe et al. 2012). From the previous Sections we have estimates of all parameters in Eq. (19), except for $\ell / a$. We can thus determine its value. We find $\ell / a=1.1$ for loop $\# 1$ and $\ell / a=1.9$ for loop \#2. The uncertainties are large and covers the whole $[0,2]$ interval for loop \#2. The uncertainty on $\ell / a$ for loop \#2 is large due to the large uncertainty in the damping time.

\section{DISCUSSION}

We have determined the Alfvén speed in a coronal loop using two independent methods: a seismological method applied to a transverse loop oscillation and a direct method taking the effects of density and magnetic stratification into account and based on magnetic potential field extrapolation and spectral information from the AIA bandpasses. We have repeated the study for two oscillating loops seen by AIA/SDO during the same flaring event, using the $171 \AA$ bandpass. For both loops, both methods give consistent results, which demonstrates that the technique of coronal seismology produces valid results. We have demonstrated that it is important to correctly interpret the observed Alfvén speed range from the oscillations. It is not sufficient to compare with an average Alfvén speed along the loop, which explains the discrepancy that Aschwanden \& Schrijver (2011) initially found.

In turn, when using a seismologically determined value of the Alfvén speed, $V_{\mathrm{A}, \mathrm{s}}$, as input for modelling or for comparison with other types of observational data, it is important to understand what this value means with respect to the Alfvén speed profile of the loop. Equation (17), where $V_{\mathrm{A}, \mathrm{d}}$ is replaced by $V_{\mathrm{A}, \mathrm{s}}$, provides a method for connecting the measurement with the Alfvén speed profile along a longitudinally stratified loop as represented by a loop top value and a scale height.

A main conclusion to draw from this study is that seismological techniques should not be seen in isolation 
from other methods. We have shown that through the formula of the Alfvén speed, the techniques of coronal seismology, magnetic extrapolation and spectroscopy are linked. Figure 7 illustrates the connections graphically. Thus, combining information from multiple techniques allows to construct more advanced methods of determining physical loop parameters or/and to build in consistency checks. We can envisage several scenarios. The obvious first scenario is the determination of the loop's magnetic field (assuming a longitudinally uniform loop) where the density from spectroscopy is combined with the Alfvén speed from seismology (Nakariakov \& Ofman 2001). When including the effect of stratification at least two parameters are required to model the Alfvén speed profile. To resolve these parameters purely seismologically, it requires a detailed measurement of the spatial wave displacement profile or of multiple wave harmonics (Andries et al. 2009). However, when this is not available, magnetic extrapolation and spectroscopy can provide alternate information. We have provided a figure (Fig. 6) to help find without calculation the expected Alfvén speed $V_{\mathrm{A}, \mathrm{d}}$ from the Alfvén speed at the loop top and the Alfvén speed scale height. Lastly, as demonstrated in Sect. 5, when combining all methods we can obtain information about the density contrast and transition layer thickness, which were impossible to determine separately by purely seismological means (Arregui et al. 2008; Goossens et al. 2008).

We have also looked at applying the same comparison between seismological and direct methods of determining the Alfvén speed to off-limb events but encountered the difficulty of obtaining an accurate value of the density due to large line-of-sight confusion. Also, the threedimensional path is less precise in that case because it is established using EUVI/STEREO as the reference (instead of the other way round for on-disk events seen by AIA/SDO). Furthermore, the magnetic extrapolation is inaccurate as it is constructed from older or later magnetogram data from near the limb.

We add the following caveats to this study. Firstly, the magnetic extrapolation is based on a potential field model that has limitations for active regions, where it is expected that free magnetic energy is stored in the field, which ultimately drives eruptions (Wiegelmann \& Sakurai 2012). Alignment between the visible loop and the magnetic field can be improved by employing force-free extrapolation models (e.g. Régnier et al. 2008) instead and using magnetic maps from HMI/SDO. Secondly, the determination of the density relied on the observation from the $171 \AA$ bandpass alone. Therefore, the determined density is only a lower limit. Ideally, we wish to obtain a better constraint on the density by using multiple bandpasses (Aschwanden et al. 2011; Hannah \& Kontar 2012) and may also incorporate density measurements from at least one loop location using spectrometer data (from e.g. EIS/Hinode, Culhane et al. 2007). Thirdly, it is critical for the three-dimensional geometry of the loop to be determined accurately. We have repeated the analysis for different loop inclination angles. A change of five degrees causes $V_{\mathrm{A}, \mathrm{d}}$, derived from extrapolation, to vary between the bottom and top range of the seismologically determined Alfvén speed range. Therefore, the derived values of loop contrast $\zeta$ and transition layer thickness $\ell$, which already have large uncertainties attached, have to be interpreted with caution.

Finally, we have not included the effect of curvature or structuring in the external corona (e.g. non-constant $\zeta$ along the loop) that may give rise to lateral wave leakage (Brady \& Arber 2005; Verwichte et al. 2006a; Van Doorsselaere et al. 2009; Pascoe et al. 2009), which modifies the relation between observed phase and kink speed as well as contributes to the observed oscillation damping. For thin coronal loops this effect is expected to be secondary.

EV acknowledges financial support from the UK Science and Technology Facilities Council (STFC) on the CFSA Rolling Grant and the SF fellowship SF/12/004 of the KU Leuven Research Council. TVD acknowledges funding from the Odysseus programme of the FWOVlaanderen and the EUs Framework Programme 7 as ERG 276808. CF acknowledges financial support from STFC under her Advanced Fellowship ST/I003649/1. RSW acknowledges support of an STFC Ph.D. studentship. AIA data is courtesy of SDO (NASA) and the AIA consortium. We thank the SOHO, STEREO and SDO instrument teams for making available data used in this paper, and Dr Marc de Rosa for useful discussions about the PFSS package.

\section{REFERENCES}

Andries, J., Goossens, M., Hollweg, J. V., Arregui, I., \& Van Doorsselaere, T. 2005, A\&A, 430, 1109

Andries, J., Van Doorsselaere, T., Roberts, B., Verth, G.

Verwichte, E., \& Erdélyi, R. 2009, Space Sci. Rev., 149, 3

Arregui, I., Ballester, J. L., \& Goossens, M. 2008, ApJ, 676, L77

Arregui, I., Andries, J., Van Doorsselaere, T., Goossens, M. \& Poedts, S. 2007, A\&A463, 333

Aschwanden, M. J., Boerner, P., Schrijver, C. J., \&

Malanushenko, A. 2011, Sol. Phys., 384

Aschwanden, M. J., de Pontieu, B., Schrijver, C. J., \& Title, A. M. 2002, Sol. Phys., 206, 99

Aschwanden, M. J., Fletcher, L., Schrijver, C. J., \& Alexander, D. 1999, ApJ, 520, 880

Aschwanden, M. J., Nightingale, R. W., Andries, J., Goossens,

M., \& Van Doorsselaere, T. 2003, ApJ, 598, 1375

Aschwanden, M. J. \& Schrijver, C. J. 2011, ApJ, 736, 102

Berghmans, D. \& Clette, F. 1999, Sol. Phys., 186, 207

Brady, C. S. \& Arber, T. D. 2005, A\&A, 438, 733

Carcedo, L., Brown, D. S., Hood, A. W., Neukirch, T., \&

Wiegelmann, T. 2003, Sol. Phys., 218, 29
Culhane, J. L., Harra, L. K., James, A. M. et al. 2007, Sol. Phys., 243, 19

Deforest, C. E. \& Gurman, J. B. 1998, ApJ, 501, L217

Dymova, M. V. \& Ruderman, M. S. 2005, Sol. Phys., 229, 79 -. 2006, A\&A, 457, 1059

Edwin, P. M. \& Roberts, B. 1983, Sol. Phys., 88, 179

Ferraro, C. A. \& Plumpton, C. 1958, ApJ, 127, 459

Goossens, M., Arregui, I., Ballester, J. L., \& Wang, T. J. 2008, A\&A, 484, 851

Goossens, M., Hollweg, J. V., \& Sakurai, T. 1992, Sol. Phys., 138, 233

Hannah, I. G. \& Kontar, E. P. 2012, A\&A, 539, A146

Hollweg, J. V. \& Yang, G. 1988, J. Geophys. Res., 93, 5423

Howard, R. A., Moses, J. D., Vourlidas, A. et al. 2008, Space Sci. Rev., 136, 67

Ionson, J. A. 1978, ApJ, 226, 650

Kliem, B., Dammasch, I. E., Curdt, W., \& Wilhelm, K. 2002, ApJ, 568, L61

Lemen, J. R., Title, A. M., Akin, D. J. et al. 2012, Sol. Phys., 275,17 
Markwardt, C. B. 2009, in Astronomical Society of the Pacific Conference Series, Vol. 411, Astronomical Data Analysis Software and Systems XVIII, ed. D. A. Bohlender, D. Durand, \& P. Dowler, 251

McEwan, M. P., Díaz, A. J., \& Roberts, B. 2008, A\&A, 481, 819 McIntosh, S. W., de Pontieu, B., Carlsson, M., Hansteen, V., Boerner, P., \& Goossens, M. 2011, Nature, 475, 477

Nakariakov, V. M. \& Ofman, L. 2001, A\&A, 372, L53

Nakariakov, V. M., Ofman, L., Deluca, E. E., Roberts, B., \& Davila, J. M. 1999, Science, 285, 862

Pascoe, D. J., de Moortel, I., \& McLaughlin, J. A. 2009, A\&A, 505,319

Pascoe, D. J., Hood, A. W., de Moortel, I., \& Wright, A. N. 2012 A\&A, 539, A37

Press, W. H., Teukolsky, S. A., Vetterling, W. T., \& Flannery,

B. P. 2007, Numerical Recipes: The Art of Scientific Computing

Régnier, S., Priest, E. R., \& Hood, A. W. 2008, A\&A, 491, 297

Ruderman, M. S. \& Roberts, B. 2002, ApJ, 577, 475

Sandman, A. W., Aschwanden, M. J., Derosa, M. L., Wülser, J. P., \& Alexander, D. 2009, Sol. Phys., 259, 1

Schmelz, J. T., Beene, J. E., Nasraoui, K., Blevins, H. T., Martens, P. C. H., \& Cirtain, J. W. 2003, ApJ, 599, 604 Schrijver, C. J. 2001, ApJ, 547, 475

Schrijver, C. J. \& De Rosa, M. L. 2003, Sol. Phys., 212, 165

Terzo, S. \& Reale, F. 2010, A\&A, 515, A7

Tomczyk, S., McIntosh, S. W., Keil, S. L., Judge, P. G., Schad, T., Seeley, D. H., \& Edmondson, J. 2007, Science, 317, 1192

Van Doorsselaere, T., Andries, J., \& Poedts, S. 2007, A\&A, 471, 311
Van Doorsselaere, T., Andries, J., Poedts, S., \& Goossens, M. 2004, ApJ, 606, 1223

Van Doorsselaere, T., Verwichte, E., \& Terradas, J. 2009, in press in Space Science Reviews

Verth, G. \& Erdélyi, R. 2008, A\&A, 486, 1015

Verwichte, E., Aschwanden, M. J., Van Doorsselaere, T., Foullon, C., \& Nakariakov, V. M. 2009, ApJ, 698, 397

Verwichte, E., Foullon, C., \& Nakariakov, V. M. 2006a, A\&A, 449,769

- 2006b, A\&A, 452, 615

Verwichte, E., Foullon, C., \& Van Doorsselaere, T. 2010, ApJ, 717,458

Verwichte, E., Nakariakov, V. M., \& Cooper, F. C. 2005, A\&A, 430, L65

Verwichte, E., Nakariakov, V. M., Ofman, L., \& Deluca, E. E. 2004, Sol. Phys., 223, 77

White, R. S. \& Verwichte, E. 2012, A\&A, 537, A49

White, R. S., Verwichte, E., \& Foullon, C. 2012, A\&A, 545, A129

Wiegelmann, T. \& Sakurai, T. 2012, Living Rev. Solar Phys., 9, 5

Wuelser, J.-P., Lemen, J. R., Tarbell, T. D. et al. 2004, in Society of Photo-Optical Instrumentation Engineers (SPIE) Conference Series, Vol. 5171, Society of Photo-Optical Instrumentation Engineers (SPIE) Conference Series, ed. S. Fineschi \& M. A. Gummin, 111-122 